\title{
Democracia, Internet Y Gobernanza: una Concreción
}

\author{
Fernando Galindo Ayuda ${ }^{1}$
}

\begin{abstract}
Resumen: El trabajo presenta una reflexión práctica sobre el alcance y los límites que tienen, desde una perspectiva jurídica, los usos democráticos en Internet, atendiendo a: 1) las afirmaciones básicas que sobre democracia se hace en los textos políticos, 2) el significado e implicaciones de algunas de las prácticas propiciadas por el uso de Internet a las que se viene caracterizando como democráticas, y 3 ) la presentación de algunas experiencias que promueven la participación de los usuarios de Internet en el conocimiento de las actividades de las instituciones públicas.
\end{abstract}

Palabras clave: Democracia. Internet. Gobernanza. Acceso a la información. Open data. Observatorio. Inclusión digital. Gobierno electrónico.

\begin{abstract}
This work presents a practical reflection, from a legal perspective, about the reaching and the limits of the democratic usage of Internet, discussing: 1) the basic affirmations made on democracy in political writings; 2) the meaning and implications of some practices propitiated by the Internet, which have been defined as democratic, and 3) the presentation of some experiences which promote the Internet users' participation in knowing the activities of the public institutions.
\end{abstract}

Key words: Democracy. Internet. Government. Access to information. Open data. Observatory. Digital inclusion. Eletronic government.

\section{Introducción ${ }^{2}$}

La expresión democracia aparece reiteradamente en Internet. Y ello no sólo (1) en lo relativo a la acumulación de información sustantiva, es

\footnotetext{
${ }^{1}$ Catedrático de Filosofía del Derecho de la Universidad de Zaragoza. E-mail: gayuda@ gmail.com.

Recebido em: 19/07/2012.

Revisado em: 30/08/2012.

Aceito em: 15/09/2012.

2 Este trabajo se desarrolla en el marco de los siguientes proyectos: Establecimiento en Iberoamérica del Observatorio de Gobierno Electrónico. EGOBS, Acción integrada para el fortalecimiento institucional, financiada por la Agencia Española para la Cooperación Internacional al Desarrollo (AECID), 2009-2012; La nueva ecología de la información y la documentación en la sociedad del conocimiento: desarrollo de una métrica sistémica, planificación de un observatorio para su seguimiento e identificación de tendencias básicas y retos estratégicos (infoscopos.com), proyecto financiado por la CICYT 2010-
} 
decir: definiciones, expresiones, teorías y conceptos, que sobre democracia se hace en la red, entendiéndola como término que ayuda a caracterizar a aquellos regímenes políticos en los que los ciudadanos eligen a sus representantes y con ello participan en el proceso de gobierno público, sino también (2) en lo que se refiere a la relación existente entre Internet y democracia, entendiendo que el funcionamiento de la misma Internet o la "red" es democrático o propicia la democracia, al considerar que facilita a todo el que la maneja, en definitiva al que cuenta con un medio de acceso a la misma un conocimiento/poder/dominio sobre la realidad que antes no lo tenía: estaba reservado a quien había sido nombrado "gobernante" en el proceso de elección democrática de representantes políticos. En el presente trabajo discurrimos sobre estas cuestiones clarificando, especialmente, el alcance del segundo de los aspectos, contrastándolo con el primero, es decir averiguando la efectiva potenciación de la democracia, entendida como participación política, que genera el uso de Internet.

A estos efectos aportamos en forma resumida, en primer lugar (2), consideraciones generalmente aceptadas sobre democracia y participación política que se producen en la actualidad, a efectos de comprender sus implicaciones. En segundo lugar (3) mostramos algunos ejemplos reales de potenciación de la democracia y de las instituciones que se ponen en práctica mediante el uso de Internet. En tercer lugar (4) recogemos algunas consideraciones sobre el hecho de que el acceso a Internet, y por su medio el acceso a información, no implica tanto el incremento de la participación política cuanto que de la comunicación. En cuarto lugar (5) nos fijamos en la relevancia de la denominada brecha digital como obstáculo a un hipotético ejercicio de la democracia participativa por los ciudadanos usando Internet. En quinto lugar (6) prestamos atención a determinadas iniciativas que potencian una efectiva realización de la democracia mediante el uso de Internet, asumiendo que democracia es, también,

2012, y Mejora en la participación en la sociedad del conocimiento a través de las actividades del Observatorio de Gobierno Electrónico. Aspectos políticos, económicos y empíricos, financiado por la Secretaría General de Universidades, Ministerio de Educación, Cultura y Deportes, convocatoria para la concesión de subvenciones para la cooperación interuniversitaria con Brasil, 2012-2013. El trabajo es un desarrollo de posiciones cuyas bases quedaron establecidas en Galindo, 2011. 
participación de ciudadanos informados en la actividad pública. En sexto lugar (7) concluimos.

\section{Democracia Hoy}

Un sistema político democrático es el que está organizado atendiendo a (1) la garantía e impulso de tres mecanismos, hoy principios jurídicos fundamentales, reconocidos en las constituciones y hechos realidad en la vida diaria de los países donde las mismas funcionan, y (2) la satisfacción de un requisito previo para el ejercicio de mecanismos y principios: el acceso a información. En el presente apartado nos referimos a estos requisitos así como (3) a la gobernanza en cuanto, como veremos, esta es la principal política o filosofía que rige hoy la puesta en práctica por los poderes públicos de mecanismos, principios y acceso a información.

\subsection{Los Principios Jurídicos Fundamentales}

Son los referidos a la libre elección de los gobernantes por los ciudadanos, la efectiva puesta en práctica de la división del ejercicio del poder político por las instancias de gobierno competentes, y la salvaguarda de la protección y promoción ${ }^{3}$ de los derechos humanos por los poderes públicos con respecto a las actividades que tienen lugar en el desarrollo de la vida propia de los ciudadanos.

Los tres mecanismos son considerados propios del funcionamiento del Estado de Derecho. Su contenido y función característicos son resumidos a continuación.

El primer mecanismo es el de la libre elección de los gobernantes por los ciudadanos, que se pone en práctica mediante la realización de procesos electorales que permiten a todos los ciudadanos participar en el gobierno nombrando periódicamente a sus representantes en distintos ámbitos políticos de decisión. Este procedimiento participativo, democrático, se ve completado por la posibilidad que tienen los ciudadanos de inter-

3 Sobre el papel activo del Estado en la promoción de derechos más que en la simple protección, véase, por ejemplo, Carbonell (2008). 
venir en las actividades gubernamentales mediante la puesta en realidad de otros medios como el referéndum con relación a cuestiones concretas sometidas a opinión de la ciudadanía por los representantes políticos, la coparticipación en actividades de carácter gubernamental mediante la intervención de ciudadanos cuya actuación está prevista en la regulación de procedimientos administrativos, y la participación de los ciudadanos en la solución de conflictos por los tribunales de justicia mediante su intervención como jurados.

El segundo mecanismo democrático es la división de poderes, es decir la aceptación de que el ejercicio de los recursos, instrumentos y medios que han de poner en práctica los gobernantes elegidos, tiene que atender a la separación de dicho ejercicio en el de tres "poderes" o funciones: legislativo, ejecutivo y judicial. Ello implica que la actividad propia de cada poder ha de ser realizada respetando la distribución de las competencias funcionales establecida mediante la satisfacción de los procedimientos reconocidos y articulados por la constitución y el resto del ordenamiento. Estos procedimientos permiten garantizar tanto la independencia de acción de cada uno de los poderes, cuanto que la coordinación y el equilibrio del ejercicio de las funciones o competencias propias de los mismos.

El tercer mecanismo es el del reconocimiento, respeto, preservación y promoción de las declaraciones de derechos humanos contenidas en la constitución y el ordenamiento jurídico en su conjunto, realizados por la práctica del ejercicio de las competencias y funciones propias de los poderes con respecto a actividades ocurridas en sociedades concretas.

\subsection{El Acceso a Información como Requisito Democrático}

Completa lo hasta aquí expresado la consideración de que, obviamente, la democracia o la participación política no puede ser puesta en práctica efectivamente en ninguna de las facetas reseñadas sin la satisfacción de un requisito previo: que los ciudadanos estén informados o, lo que es lo mismo, tengan suficiente conocimiento sobre el objeto de su participación. 
Es por ello que en la actualidad cabe decir, sintéticamente, que un sistema político democrático es aquel cuyo funcionamiento está basado en la participación consciente e informada de los ciudadanos en el ejercicio del poder político o bien indirectamente mediante la elección de sus representantes o bien directamente colaborando en la toma de decisiones políticas utilizando otros mecanismos. Esto implica reconocer que los ciudadanos pueden participar en prácticamente todas las actividades de los poderes públicos, atendiendo, además, al hecho de que el Estado de Derecho actual no es el Estado liberal del siglo XIX que limitaba la acción de los organismos públicos a actuar políticamente como policía elaborando las correspondientes leyes básicas y aplicándolas mediante penas o sanciones a los infractores del orden jurídico: salvaguardando el funcionamiento del mercado, sus posibles violaciones, sino que el Estado es, a la vez, Estado social, democrático, del bienestar, de la gobernanza y, hoy, el Estado propio de la denominada sociedad del conocimiento, que tiene potestad para participar en prácticamente todas las actividades diarias, especialmente las propias de las instituciones públicas una vez están financiadas con fondos públicos.

\subsection{Gobernanza}

A lo dicho hasta aquí no le impide el reconocimiento que de un tiempo a esta parte se está aceptando como práctica política propia común de las competencias propias de los poderes públicos la del ejercicio de lo que se denomina gobernanza. La gobernanza está definida por el Diccionario como "Arte o manera de gobernar que se propone como objetivo el logro de un desarrollo económico, social e institucional duradero, promoviendo un sano equilibrio entre el Estado, la sociedad civil y el mercado de la economía".

Lo anterior implica reconocer la expansión en el ámbito público, como prácticas o usos propios de los gobernantes (incluyendo en la expresión a todos los funcionarios públicos que ponen en acción a los tres poderes), de los principios, técnicas o usos de gobierno propios del ámbito empresarial. Esto es lo mismo que decir: la puesta en acción de la 
eficiencia y las reglas del mercado como criterio de acción preferente de los poderes públicos.

Este estilo de acción o política no impide lo expresado en los anteriores apartados, es decir la circunstancia de que ha de ser ejercido por los poderes en forma compatible con la puesta en práctica de los principios propios del Estado de Derecho, que resumen la acción de la democracia que, por mandato legal, gobierna la acción de los poderes públicos, es decir todos aquellos asuntos sobre los que éstos son competentes según el ordenamiento propio de los Estados de Derecho en cuanto son agentes activos en la vida social y política de la sociedad del conocimiento ${ }^{4}$. Ello se predica especialmente de la aplicación del Derecho, realizada por los juristas, según se reconoce genéricamente, en el proceso judicial de forma compleja: atendiendo al mecanismo de la ponderación, propio de la gobernanza, más que a la aplicación "automática" de la subsunción. Es conveniente resumir a estos efectos el mensaje básico enviado por algunos de los estudiosos, filósofos del Derecho, de las decisiones judiciales a prácticos del Derecho desde un tiempo ya lejano como el constituido por los comienzos del siglo $\mathrm{XX}^{5}$.

Desde aquella época, justamente desde el comienzo de la obligación de los jueces de poner en ejecución el Código Civil alemán bajo su responsabilidad ante todos los casos que los ciudadanos les plantearan, surgieron consideraciones críticas con respecto a la idea de que la aplicación del Derecho por los jueces estaba reducida a la realización de la subsunción del caso concreto en la Ley, como planteaban y presumían los Códigos liberales. Ehrlich, junto a los tratadistas y jueces que se integraron en el Movimiento de Derecho Libre, puso de manifiesto que el proceso de aplicación del Derecho no podía estar reducido a la subsunción una vez que la irremediable existencias de lagunas jurídicas hace que la mayor parte de las resoluciones judiciales sean creaciones "libres", de los mismos jueces, a efectos de no incurrir en la responsabilidad corres-

4 Se hacen propuestas a este respecto en relación al ámbito local en Estados Unidos en: Katz (2010). Me he referido a la conciliación entre gobernanza y derecho en: Galindo (2007).

5 Informa sobre el estado de la cuestión de la discusión filosófico jurídica sobre estas cuestiones: Robles (2010). 
pondiente por no tomar decisiones en casos, sometidos a su decisión por imperativo legal, cuyos supuestos y soluciones no coincidieran con los previstos por la Ley. (EHRLICH, 1903)

A partir de estas consideraciones surgieron a lo largo del siglo $\mathrm{XX}$ hasta la actualidad múltiples reflexiones dirigidas a completar el proceso de aplicación judicial del Derecho con otras explicaciones. Algunas de las soluciones propuestas fueron las siguientes: el conocimiento de las concepciones y convicciones sociales (propuesta hecha por Ehrlich a través de la Sociología: el derecho vivo), la consideración de que el proceso judicial y el razonamiento jurídico están integrados por tópicos o lugares comunes que auxilian a la aplicación (VIEWEG, 1974), el establecimiento de sistemas normativos auxiliares a la aplicación elaborados mediante el uso de la lógica contando con la construcción de la pirámide normativa que amplía racionalmente el ámbito legal (KELSEN, 1979), la propuesta del estudio de las leyes atendiendo a que se interpretan a partir de la “precomprensión” de su contenido (ESSER, 1961), Engisch (1968) y Gadamer (1977), el estudio de la aplicación judicial del Derecho atendiendo al amplio ámbito y contenido de las argumentaciones que en la misma se produce (PERELMAN, 1979; ALEXY, 1992), la consideración del acuerdo de legitimación social: el consenso, al que están dirigidas las leyes y la organización estatal en su totalidad (los tres poderes) en las sociedades democráticas (HABERMAS, 1998), la consideración de que todas las actividades humanas son realizadas atendiendo a un conocimiento de la realidad producido en el contacto mantenido con la misma realidad: "autopoiéticamente" (MATURANA, 1990), y no por la mera elucubración o desarrollo intelectual de las propuestas científicas.

Estas y otras propuestas estaban ocupadas, resumiendo, en poner énfasis en el contexto propio de la aplicación judicial, a efectos de explicarla y darle soluciones más complejas que las que establece la subsunción o el discurrir formal sobre los textos jurídicos. 


\section{TIC y Democracia}

No cabe duda de que las Tecnologías de la Información y la Comunicación (TIC) e Internet facilitan la puesta en funcionamiento de los sistemas políticos democráticos. Aquí vamos a presentar tres ejemplos que lo prueban inequívocamente. Uno está referido al auxilio a la elección de los representantes políticos. Otro se refiere al acceso de los ciudadanos a los servicios públicos por medios electrónicos. El último se ocupa del apoyo al funcionamiento del poder judicial. A continuación resumimos las características básicas de los ejemplos.

El primer ejemplo tiene manifestaciones masivas en Brasil, por ejemplo. Desde la segunda mitad de los años noventa los procesos electorales destinados al nombramiento de los representantes políticos son auxiliados por el uso de las denominadas "urnas electrónicas", programas y ordenadores que facilitan que el derecho de voto con el fin de elegir a sus representantes políticos que tienen todos los ciudadanos sea ejercido por la selección por los electores de los candidatos mediante la pulsación, autorizada por los integrantes de la mesa de votación a los electores que son identificados mediante la aportación de un documento, del número correspondiente, la denominación, asignado a los candidatos a ser elegidos. El programa almacena las opciones tomadas por los electores y suma los resultados finales de la votación. De esta forma el uso de las tecnologías mejora el ejercicio del voto al dificultar la realización de prácticas corruptas electorales que en Brasil permitían los sistemas tradicionales de elección política, promoviendo con ello el uso de las TIC la ampliación y profundización de la democracia ${ }^{6}$.

El segundo ejemplo está extendido por muchos países. Es el denominado Gobierno electrónico o, más adecuadamente, Administración electrónica ${ }^{7}$. Hace referencia a la provisión por medios electrónicos de

\footnotetext{
6 Véase al respecto: Mezzaroba y Rover (2009).

7 El plan de gobierno electrónico de la Unión Europea para el período 2011-15 está regulado en: Communication from the Commission to the European Parliament, the Council, the European Economic and Social Committee and the Committee of the Regions, The European eGovernment Action Plan 2011-2015. Harnessing ICT to promote smart, sustainable \& innovative Government (2010), COM/2010/0743 final. Recuperado el 14
} 
acceso de los ciudadanos a los servicios públicos. Implica que los ciudadanos puedan tramitar por Internet desde sus domicilios o sus propios ordenadores instancias a las Administraciones Públicas sin necesidad de realizar desplazamientos a la sede de las Administraciones de las que se requiera la satisfacción de un derecho concreto. El proceso tiene lugar de la siguiente forma. Una vez identificado fidedignamente el solicitante mediante la utilización de una clave obtenida usando un procedimiento seguro, el ciudadano interesado puede remitir por Internet su solicitud, junto al expediente que la justifica o fundamenta, al ordenador-programa de la Administración, la sede electrónica, cuya sección administrativa competente tiene la obligación de tramitar el expediente reconociendo lo requerido por el solicitante. El sistema tecnológico puede mejorar, por tanto, la calidad democrática de la Administración correspondiente, al incrementar la prontitud de respuesta de los servicios que la integran, potenciando de esta forma la satisfacción de los derechos de los ciudadanos ${ }^{8}$.

El tercer ejemplo está referido al ámbito judicial y sucede en varios países. Por ejemplo9. Un abogado, convenientemente identificado, puede enviar a un Juzgado o Tribunal desde cualquier ordenador un documento que forme parte de un proceso en el que esté implicado un ciudadano defendido (o acusado) por el abogado, a efectos de que el trámite procesal se dé por realizado pudiéndose con ello continuar su tramitación en un periodo de tiempo menor que el que es preciso para que el procedimiento discurra por los canales establecidos para la tramitación del documento en formato papel al evitarse las dilaciones propias del funcionamiento del correo ordinario. El sistema, además, permite a todas las partes implicadas en el proceso comprobar el estado de tramitación del procedimiento,

de marzo de 2012 en: < http://eurlex.europa.eu/LexUriServ/LexUriServ.do?uri=CELEX: DKEY=548503:EN:NOT $>$.

8 Sobre servicios administrativos en línea para ciudadanos en España véase: $<$ http:// www.060.es/060/appmanager/portal/desktop/page/ciudadanosHome>. Recuperado el 14 de marzo de 2012.

9 Se toma como referencia el funcionamiento del sistema español LEXNET: "sistema informático de telecomunicaciones Lexnet para la presentación de escritos y documentos, el traslado de copias y la realización de actos de comunicación procesal por medios telemáticos", regulado por el Real Decreto 84/2007, de 26 de enero. La página web del sistema LEXNET es: < http://infolexnet.justicia.es/>. Recuperado el 14 de marzo de 2012. 
pudiéndose conocer tanto las fases del proceso satisfechas cuanto el contenido de los documentos presentados por la otra parte o las resoluciones o diligencias adoptadas por el órgano instructor o juzgador. Las TIC favorecen también en este caso la democracia una vez que el procedimiento que facilitan permite tanto acortar los tiempos precisos para el ejercicio de las labores propias del poder judicial: la resolución de conflictos, cuanto hacer más transparentes las fases de tramitación del procedimiento, permitiendo conocer por las partes implicadas en el caso el estado en el que la tramitación se encuentra, sin que por ello pierda el proceso judicial las funciones conferidas al mismo por el ordenamiento democrático.

No cabe duda de que estos procesos son ejemplos de que las TIC son efectivos instrumentos auxiliares del funcionamiento de los sistemas democráticos.

A efectos de explorar otras posibilidades discurrimos en el resto del trabajo sobre si las TIC permiten:

- auxiliar al proceso de elección de los representantes políticos, además de completar la mecánica de puesta en práctica del proceso electoral en algunos aspectos problemáticos del mismo, y

- mejorar la participación de los ciudadanos en la puesta en acción del Estado de Derecho permitiéndoles intervenir en decisiones políticas con un mayor y mejor conocimiento de la realidad política y su funcionamiento que el que tienen sin el uso de las TIC.

\section{Democracia e Internet}

Ha quedado reseñado al comienzo de este trabajo que la expresión democracia se utiliza con gran frecuencia como vocablo caracterizador de Internet. Se dice que promueve la participación; que presenta a la opinión pública: a los ciudadanos, información que antes se encontraba sólo en poder de organismos gubernamentales o partidos políticos; que permite expresar a todos opiniones ante cualquier asunto que presenten en la red Gobiernos, particulares, asociaciones o empresas. 
En este apartado presentamos algunos de los límites que cabe hacer a este tipo de afirmaciones. En concreto nos centramos en:

- mostrar las características básicas de los usos que se hace de Internet en estos momentos; y

- reseñar algunas de las características de la gobernanza de Internet, especialmente en lo relativo a la posibilidad de manifestar opiniones libre y secretamente.

\subsection{Internet y Promoción de la Democracia}

A efectos de considerar datos concretos nos fijamos en información referida al uso de Internet en dos ámbitos: porcentaje de casas o domicilios con acceso a Internet y porcentaje de declaraciones de usos de las aplicaciones de Internet.

\subsubsection{Domicilios}

No cabe negar el elevado grado de acceso a Internet que se produce en estos momentos. Acudiendo a estadísticas oficiales (EUROSTAT), estas dan cuenta de que la cifra promedio de viviendas con acceso a Internet en Europa en 2011 es del 73 por ciento de las mismas, frente al 49 por ciento de 2006. El porcentaje todavía aumenta más si se considera el número de viviendas con acceso a Internet en las que viven niños: llegaba al 84 por ciento en 2010 , mientras que el promedio de viviendas con acceso a Internet en las que no viven niños alcanzaba al 65 por ciento (en 2010) ${ }^{10}$.

\subsubsection{Aplicaciones Usadas}

Existen también datos estadísticos sobre el tipo de las aplicaciones, programas o usos que se hace de Internet. Según dichos datos se utiliza Internet básicamente para realizar los siguientes tipos de comunicaciones:

1. correo electrónico;

\footnotetext{
10 Vease al respecto los resultados de la encuesta titulada "Households with Internet access at home": <http://epp.eurostat.ec.europa.eu/tgm/refreshTableAction.do?tab=table \&plugin=1\&pcode=tin00088\&language=en $>$. Recuperado el 13 de marzo de 2012.
} 
2. envío de mensajes a programas - páginas web- que facilitan la realización de conversaciones simultáneas ("chats") y publicación de noticias ("blogs"); y

3. realización de llamadas telefónicas y videoconferencias ${ }^{11}$.

Es lógico considerar que cuando nos ocupamos de democracia sólo las comunicaciones referidas en segundo lugar: el envío de mensajes a programas o páginas web, pueden ser tomadas como referencia para discurrir sobre un posible ejercicio de la democracia utilizando Internet. Recuérdese que, tal y como vimos en el primer apartado (2.1), democracia es lo mismo que emisión de opiniones con relevancia pública o "participación de los ciudadanos en el ejercicio del poder político o bien indirectamente mediante la elección de sus representantes o bien directamente colaborando en la toma de decisiones políticas utilizando otros mecanismos". Los otros usos, tanto el intercambio de correos electrónicos, como las conversaciones telefónicas o las videoconferencias, están referidos a comunicaciones privadas habidas entre los interlocutores que los utilizan.

Considerando los datos estadísticos, es interesante comprobar, contemplando las cifras recopiladas sobre uso de Internet en Europa ${ }^{12}$, que hay una diferencia sustancial en el porcentaje de usuarios que en 2010 utilizaron Internet para realizar "chats", enviar "blogs" y participar en actividades de redes sociales: 75 (entre 16 y 24 años), 33 (entre 25 y 54) y 7 (entre 55 y 74); el promedio de uso de estas herramientas fue el 32 por ciento de la totalidad de los usuarios. La diferencia es más destacable si se tiene en cuenta que frente a estos datos el promedio general de individuos que en Europa utilizaron en 2010 Internet para enviar y recibir mensajes

\footnotetext{
11 El promedio europeo de realización de llamadas telefónicas y videoconferencias por internet por personas era muy bajo: 10 por ciento en 2007 cuando se realizó la encuesta. Ver: "Internet activities - Individuals: Internet use: telephoning, videoconferencing": $<$ http://appsso.eurostat.ec.europa.eu/nui/show.do?dataset=isoc_ci_ac_i\&lang=en $>$. Recuperado el 14 de marzo de 2012.

12 "Individuals using the Internet for posting messages to social media sites or instant messaging. Posting messages to chat sites, social networking sites, blogs, newsgroups or online discussion fora or use of instant messaging": <http://epp.eurostat.ec.europa. $\mathrm{eu} / \mathrm{tgm} /$ refreshTableAction.do?tab=table\&plugin $=0 \&$ pcode $=$ tin $00084 \&$ language $=\mathrm{en}>$. Recuperado el 13 de marzo de 2012.
} 
por correo electrónico en 2010 fue mucho más superior: prácticamente el doble, el 61 por ciento $^{13}$.

\subsubsection{Conclusiones Sobre el Uso de Internet y Democracia}

Los datos hasta aquí expuestos nos permiten concluir lo siguiente.

Si bien es indudable la fuerte expansión alcanzada por Internet, el promedio del acceso a la red es del 73 por ciento de los domicilios europeos, ello no permite, hipotéticamente, por ejemplo, la aprobación de una norma de ámbito europeo que establezca la obligación de elegir a los representantes políticos usando las TIC: desde los propios ordenadores. Para que esta norma pudiera entrar en vigor debería ser posible un acceso del cien por cien de la población, lo que no se produce en la actualidad.

La expansión de la democracia tampoco es viable absolutamente en estos momentos si tenemos en cuenta las características de los usos de Internet. Como hemos visto tan sólo el sector más joven de la población: el comprendido entre los 16 y los 24 años, utiliza mayoritariamente sistemas que permiten expresar opiniones (el 75 por ciento de ellos atendiendo a las cifras generales europeas) mientras que los otros dos sectores entre 25 y 54 y entre 55 y 74 los usan en escasa medida ( 33 y 7 por ciento respectivamente).

\subsection{La Gobernanza de Internet}

Existe otro problema de mayor entidad que el del reducido número de usuarios que acostumbra a emitir opiniones usando la red que acabamos de señalar como límite para la expansión de la democracia por Internet. El problema de mayor envergadura está ligado a las características del funcionamiento de Internet y su regulación, que impiden garantizar el secreto de las opiniones emitidas, principio que, entre otros, requiere la puesta en acción de los procesos democráticos. A este problema lo identificamos como la gobernanza de Internet.

\footnotetext{
13 "Internet use and activities: sending/receiving emails" <http://appsso.eurostat. ec.europa.eu/nui/show.do?dataset=isoc_ci_ac_i\&lang=en $>$. Recuperado el 13 de marzo de 2012 .
} 
Con gobernanza de Internet nos referimos a las reglas básicas de funcionamiento del sistema, que, más allá del uso de las tecnologías, vienen a ser, con algunas moderaciones, las mismas que las del mercado o la obtención de beneficios.

En efecto. La utilización de Internet requiere, indefectiblemente, al ciudadano el pago de su uso: básicamente a la compañía de telecomunicaciones que garantiza la comunicación y al proveedor de servicios de acceso a Internet que permite la "navegación", también a los que ofertan servicios "accesibles" através de la red. El último pago se hace de una forma indirecta: o bien al abonar los precios de los bienes adquiridos una vez que en dicho precio están repercutidos los costes de comercialización y envío del producto hechos por el vendedor utilizando Internet, o bien, en el caso del acceso a servicios públicos, al pagar los impuestos y tasas al Estado, una vez que parte de ellos sirve para satisfacer el coste de los servicios que son accesibles y tramitados por Internet.

A lo anterior ha de añadirse otra faceta o dimensión, si tomamos en consideración la hipótesis de la utilización de Internet como canal para ejercer la democracia en el proceso de elección de gobernantes o mediante la emisión de opiniones en referéndum sobre un tema sometido a decisión de los ciudadanos por las instituciones públicas. La nueva dimensión procede de la circunstancia de que si bien, como ya veíamos en los ejemplos mencionados más arriba (3) referidos al acceso a los servicios de las Administraciones públicas o a la tramitación de expedientes judiciales, el ejercicio de dicho acceso requiere la inequívoca identificación de quien emite su opinión, tal y como sucede en la solicitud de servicios públicos o en la actuación en la Administración de justicia: ha de saberse quién ejerce su derecho y que lo hace en un momento concreto, mientras que la actuación de la democracia por medio de las elecciones, en cambio, requiere algo más: garantizar y preservar el secreto de las opiniones emitidas a efectos de que estas sean hechas en libertad (las exigencias del voto son: sufragio universal, libre, igual, directo y secreto). Y aquí es dónde la relación entre democracia e Internet tiene tantas dificultades que cabe decir, incluso, que por el momento esa relación no es posible ni siquiera contando con recursos tecnológicos como enfatizamos a continuación. 
Tanto la procuración electrónica de servicios públicos como la remisión de documentos a instancias judiciales requiere garantizar la autenticidad del remitente, la del proceso de comunicación o el envío de los documentos: que no sea interceptado, y la de que el contenido de lo enviado sea lo efectivamente remitido por el emisor, lo que sucede mediante la utilización de los requisitos técnicos expuesta resumidamente más arriba, sin que dichos procedimientos requieran mantener permanentemente el secreto del contenido del comunicado. Es más: en la tramitación de procedimientos administrativos y judiciales es preciso, básicamente, poder evidenciar a lo largo de todo el procedimiento que el contenido de los documentos comunicados es el refrendado por el remitente.

El requisito fundamental para garantizar el funcionamiento del proceso de emisión de una opción u opinión política, en cambio, es el de la preservación permanente del secreto de la opción u opinión del responsable, de otra forma los electores u opinantes no pueden contar con suficiente libertad como para emitir su voto u opción, otro principio básico democrático. El problema reside en que si bien la identificación fidedigna es posible, como ocurre en los ejemplos mencionados en el apartado (3), mediante la utilización de sistemas TIC de identificación, de coste mayor o menor, la garantía de la preservación del secreto y de la emisión libre de las opiniones no es posible ponerla en práctica sino, a lo sumo, mediante la utilización de mecanismos como el cifrado de clave pública de confidencialidad, de uso general no autorizado en estos momentos por razones de seguridad ${ }^{14}$.

Es decir: en el caso de las elecciones por Internet el principio de seguridad pública establece fuertes límites al principio de mercado o de las reglas de gobernanza que rige el funcionamiento propio de Internet, y que es el que delimita en estos momentos, básicamente, la práctica relativa a

14 Sobre los pros y contras de las “elecciones electrónicas” ver: Alvarez y Hall (2010). Se establecen indicaciones por el Consejo de Europa sobre los requisitos para poner en funcionamiento a la democracia electrónica en forma respetuosa con los principios democráticos en la Recommendation CM/Rec (2009) of the Committee of Ministers to member states on electronic democracy (e-democracy) (Adopted by the Committee of Ministers on 18 February 2009 at the 1049th meeting of the Ministers' Deputies), ver al respecto: Council of Europe (2009). 
la libre expresión de ideas que se manifiesta entre los usuarios de Internet mediante el uso de instrumentos de comunicación como el acceso a las redes sociales, el envío de noticias a los "blogs" o el intercambio de mensajes utilizando sistemas de correo electrónico, comunicación telefónica o videoconferencia.

\section{Uso de Instrumentos Técnicos y Brecha Digital}

Es conveniente reconsiderar la información que suministran los datos estadísticos para tener una mayor conciencia sobre las posibilidades e implicaciones que tiene un hipotético intento de realizar un ejercicio de la democracia, entendida como ejercicio del sufragio político, utilizando las herramientas y mecanismos que proporciona Internet.

En el apartado anterior hemos observado que no es posible realizar elecciones políticas por medio de Internet por varias razones, en este apartado vamos a matizar todavía más el alcance de los argumentos mostrando que cuando pasamos de considerar los promedios estadísticos generales referidos a un amplio espectro de la población a atender a los relativos a determinados países las cifras tienen contenidos, y significación, distintos.

Fijémonos en las cifras referidas al porcentaje de viviendas con acceso a Internet. Decíamos que el acceso promedio en Europa era alto: el 73 por ciento de las casas lo tenía en 2011 , y 84\%, ya en 2010, era el promedio de las casas con niños. Las cifras alcanzan distinto cariz si nos fijamos en España cuyos porcentajes son, respectivamente, 64 y 73, Alemania, 83 y 97 o Rumanía, 47 y 50, por ejemplo ${ }^{15}$. El matiz todavía es más importante si nos fijamos en Suramérica. En Brasil, en 2010, sólo el 27 por ciento de las casas contaba con acceso a Internet, siendo el mismo número promedio el del 31 por ciento en las viviendas situadas en ámbito urbano y el $6 \%$ en las viviendas situadas en ámbito rural ${ }^{16}$.

15 Ver: los resultados de la encuesta titulada "Households with Internet access at home": $<$ http://epp.eurostat.ec.europa.eu/tgm/refreshTableAction.do?tab=table\&plugin=1\&pco de $=$ tin00088\&language $=$ en $>$. Recuperado el 14 de marzo de 2012.

16 Ver: "PROPORÇÃO DE DOMICÍLIOS COM ACESSO À INTERNET" < http://www. cetic.br/usuarios/tic/2010-total-brasil/rel-geral-04.htm>. Recuperado el 14 de marzo de 
Estos datos dan cuenta de la existencia efectiva de la denominada brecha digital y de cómo la reflexión sobre la posibilidad de votar a través de Internet es un discurso estrecho: de limitado alcance social inmediato, necesita ser completado. Pensemos en que una hipotética puesta en práctica inmediata que requiriera su uso obligatorio en un país implicaría privar del derecho de voto a un ingente número de personas.

\section{Acceso a Información}

Expresábamos al comienzo de este trabajo (2.2) que un requisito fundamental para el ejercicio de la democracia es contar con información suficiente para decidir. Ello es coherente con el hecho de que sin información no es posible tomar decisión alguna ${ }^{17}$. Si esto es así en las actividades propias: en el ejercicio de la libertad o del principio de autonomía de la voluntad, la misma o mayor relevancia tiene en el ámbito de acción de los hombres referido a las actividades que implican a otros hombres. Especialmente en la acción política y el ejercicio democrático de la libre elección de los representantes políticos: no se puede elegir sin conocer previamente las opciones sobre las que cabe optar.

E Internet proporciona, sin duda, acceso a información. Ofrece una información que antes, sin la red, no estaba al alcance de los ciudadanos. La información quedaba, a lo sumo, recogida en documentos: estudios, informes, artículos científicos y de opinión, libros y noticias suministrada por los medios de publicación, opinión e información: periódicos, revistas y otros medios (televisión y radio fundamentalmente). Otra parte de la

2012. Los datos son recopilados por el brasileño "Centro de Estudos sobre as Tecnologias da Informação e da Comunicação" (CETIC.br)

17 Sin establecer contactos con el exterior. Ver al respecto Maturana, 2006. En este artículo se muestra el origen de la auto-consciencia, mostrándola como un suceder que ocurre en el "lenguajear", y no confundiendo los dominios en los cuales ocurre el vivir, ni reduciéndolos. Para esto se basa en los fundamentos biológico-culturales de lo humano, buscando siempre el mecanismo estructural-operacional que da origen al fenómeno por el cual se pregunta sin introducir nociones semánticas. El autor también reflexiona sobre las consecuencias y alcances de este mirar, esto es, que la autoconsciencia no ocurra en el cerebro y que no sea una entidad física sino que está ocurriendo como una configuración de sentires (sensorialidades) en un presente en continuo cambio. 
información y especialmente la referida a las actividades del Estado quedaba fuera de los círculos de opinión. Con gran frecuencia se consideraba secreta: estaba a disposición únicamente de las instituciones estatales.

La información necesaria para elegir democráticamente es la referida a las actividades de los representantes políticos, cuya expresión se manifiesta en la acción propia de las instituciones en las que los mismos las realizan. Son las actividades de los poderes legislativo, ejecutivo y judicial. Están recogidas en leyes, normas, reglamentaciones, sentencias y, ahora, también en páginas web en las que se presenta tanto el contenido de las regulaciones cuanto que informaciones sobre las características y resultados de las actividades desenvueltas y los servicios públicos ofertados por cada institución.

La información relativa al contenido de las regulaciones es la misma, sobre formato digital, que la que se hacía pública en papel. La principal diferencia resulta del grado de expansión de la información digital: es mayor que la que tiene la recogida en formato papel. La información sobre las instituciones, sus actividades y servicios, es de diferente entidad: tradicionalmente no era accesible, no existía o a lo sumo encontraba algún eco en los medios de comunicación. En la mayor parte de las ocasiones estaba expresada en el contenido de los propios textos jurídicos o regulaciones. En la actualidad esta información es generada por la propia institución, que puede ofrecer sus servicios a los ciudadanos a través de Internet, como recogíamos más arriba (apartado 3).

Esta información precisa ser expuesta, publicitada y recopilada de forma ordenada y suficientemente clara si se quiere que los ciudadanos puedan acceder a la misma, utilizar los servicios públicos y realizar el control democrático de la misma que compete a los ciudadanos. Paso previo es el establecimiento de algunas indicaciones para efectuar adecuadamente esta recopilación, atendiendo tanto al contenido de las prácticas desarrolladas como a lo que prescriben las reglas de la democracia encaminadas a potenciar la participación de los ciudadanos en el poder político. En España no se ha promulgado una Ley de transparencia y acceso a la información pública que es la que debe dar indicaciones generales sobre la información a publicar. La única regulación existente, por ahora, 
es la Ley 37/2007, de 16 de noviembre, sobre reutilización de la información del sector público, para el ámbito del sector público estatal y el Real Decreto 1495/2011 que desarrolla la Ley ${ }^{18}$. Esta normativa es insuficiente al no estar desarrollado el marco general el gobierno y las instituciones públicas no están obligadas a facilitar a los ciudadanos la información necesaria para tomar decisiones importantes en el ámbito personal, profesional y político. Es por ello que hasta ahora lo que existen son iniciativas que tienen por objeto hacer llegar a los ciudadanos información pública: "open data", que se encuentra en Internet ${ }^{19}$.

La más mínima observación sobre la información recopilada por este tipo de iniciativas ${ }^{20}$ da cuenta de que responde a fines u objetivos dispares: ofertar la información a elaboradores de estudios, disponer acceso seguro a servicios públicos, mostrar el alcance de las iniciativas administrativas propias de la entidad administrativa responsable de la página o sitio web, señalar los avances de la técnica, poner en funcionamiento el principio legal de transparencia de las actividades gubernamentales, mostrar el grado de eficiencia de políticas de gobernanza, generar actividades a desarrollar por empresas y con ello crear empleo. Todo lo cual trae como consecuencia la existencia de numerosa información en Internet que está situada, todavía, en forma escasamente accesible al ciudadano, una vez que éste no cuenta con suficientes conocimientos ni recursos como para poder acceder a la información ni, una vez accedida, compren-

18 Se puede acceder a la Ley en <http://www.boe.es/diario_boe/txt. php?id=BOE-A-2007-19814>. Al Real Decreto en: < http://www.boe.es/diario_boe/txt. php?id=BOE-A-2011-17560 $>$. Ponen en práctica la Directiva europea sobre transparencia: Directiva 2003/98/CE del Parlamento Europeo y del Consejo de 17 de noviembre de 2003 relativa a la reutilización de la información del sector público. $<$ http://eurlex.europa.eu/ LexUriServ/LexUriServ.do?uri=CELEX:32003L0098:ES:HTML>.

19 Elaborados por el Gobierno del País Vasco: http://opendata.euskadi.net/w79-home/ es. Sobre actividades administrativas del Gobierno del Reino Unido: http://data.gov.uk/. La Fundación sobre datos abiertos, organización no lucrativa, promueve el uso de datos estadísticos: < http://www.opendatafoundation.org $>$. Las páginas web han sido accedidas el 15 de marzo de 2012.

20 Vease la información contenida en iniciativas gubernamentales españolas sobre "open data" como APORTA (<http://www.aporta.es/web/guest/news.consulok>, consultado el 14 de marzo de 2012) y Datos.gob.es (<http://datos.gob.es/datos/>, consultado el 14 de marzo de 2012). 
derla. Ello, por tanto, no se debe únicamente al limitado porcentaje de personas que acceden a la red del que se daba cuenta en las estadísticas recogidas en apartados anteriores, la brecha digital, sino al hecho de que la información está colocada con gran frecuencia en lenguaje propio de profesionales expertos en contabilidad, por ejemplo, pero no en lenguaje comprensible por los ciudadanos.

Es por ello que de un tiempo a esta parte se producen otras iniciativas que tienen el objetivo de formar a profesionales y promover la participación ciudadana en forma activa en el ejercicio de sus derechos democráticos, haciendo accesible mediante Internet a los ciudadanos el mismo funcionamiento y actividades de las Administraciones públicas y de los diferentes poderes públicos.

En este marco de acción se encuentran las investigaciones y desarrollos que desde 2003 se realizan por el Observatorio Internacional de Gobierno electrónico.

El objetivo fundamental del Observatorio EGOBS (Electronic Government Observatory) es el estudio independiente de las características de las realizaciones que en materia de Gobierno electrónico tienen lugar en distintos países.

Los trabajos realizados ponen especial énfasis en la comprobación referida a si la prestación se realiza con respeto a las normas, procedimientos y principios recogidos en las leyes, incluido el derecho a la participación en dichas actividades de ciudadanos, empresas e instituciones $\mathrm{y}$, especialmente, si se satisface en su puesta en práctica las regulaciones sobre protección de datos y seguridad de las comunicaciones electrónicas.

EGOBS (www.egobs.org) ${ }^{21}$ es una iniciativa surgida de la red temática Gobierno Electrónico coordinada por la Universidad de Zaragoza (España) y en cuyas actividades participan desde 2003 las Universidades de Burgos y Valladolid (España), de Münster (Alemania) y la Queen's

\footnotetext{
${ }^{21}$ La página ha tenido 215.387 visitas entre el 1.1.2007 y el 15.3.2012. Los visitantes procedían de 147 países/regiones. Los diez primeros países por número de visitas (de mayor a menor) son: Estados Unidos, China, Chile, España, Méjico, Uruguay, Federación Rusa, Argentina, Venezuela y Perú. Los informes sobre visitas han sido elaborado teniendo en cuenta la información que recopilar el programa URCHIN de Google.
} 
University de Belfast (Reino Unido), por parte europea. Los miembros americanos son la Universidad Nacional de la Plata (Argentina), la Diego Portales de Santiago de Chile, la de la Habana (Cuba) y la de la República de Montevideo (Uruguay). El Observatorio es parte de la Red Jurídica para la Sociedad de la Información LEFIS (Legal Framework for the Information Society) $)^{22}$.

Desde el 1 de enero de 2009 el Observatorio cuenta con infraestructura personal y material para el desarrollo de sus actividades en Iberoamérica. En concreto en la Universidad Federal de Santa Catalina (Brasil) ${ }^{23}$.

Las actividades de observación de EGOBS han estado centradas en aplicar, con fines docentes e investigadores, una metodología desarrollada para estudiar páginas web, confeccionadas e implementadas por Administraciones públicas, utilizando criterios de análisis que permiten conocer y presentar públicamente las características de las propias páginas, sus contenidos y el de las ofertas de servicios que realizan las Administraciones que las han confeccionado y mantienen ${ }^{24}$.

La metodología se ha venido aplicando desde el año 2005 a páginas web desarrolladas por instituciones públicas en Chile, Uruguay, España y Brasil, fundamentalmente. Los resultados de los análisis están expuestos en Internet ${ }^{25}$.

22 La página ha tenido 1.071.805 visitas entre el 1.1.2007 y el 15.3.2012. Los visitantes procedían de 204 países/regiones. Los diez primeros países por número de visitas (de mayor a menor) son: Estados Unidos, China, España, Japón, Letonia, Alemania, Federación Rusa, Reino Unido, Francia y Brasil. Los informes sobre visitas han sido elaborado teniendo en cuenta la información que recopilar el programa URCHIN de Google.

$23<$ http://www.egov.ufsc.br/portal/>. La página ha sido accedida el 15 de marzo de 2012. La página ha tenido 1.092.775 visitas entre el 1.1.2011 y el 15.3.2012. Los visitantes procedían de 174 países/regiones. Los diez primeros países por número de visitas (de mayor a menor) son: Brasil. Estados Unidos, China, Portugal, Reino Unido, Japón, Francia, España, Alemania y Mozambique. Los informes sobre visitas han sido elaborado teniendo en cuenta la información que recopilar el programa URCHIN de Google.

24 La métrica LEFIS. Ver su contenido en: < http://www.egobs.unizar.es/index. php?option $=$ com_content $\& \% 2520$ task $=$ view\&id=14\&Itemid $=27>$. La página ha sido accedida el 15 de marzo de 2012.

25 Ver: $<$ http://www.egobs.unizar.es/index.php?option=com_content\&task=view\&id=2 $1 \&$ Itemid $=64>$ y $<$ http://www.egobs.unizar.es/index.php?option=com_content\&task=vi 
A partir de 2009 EGOBS realiza en la misma línea otro tipo de análisis destinado a presentar en Internet a ciudadanos en forma clara la localización geográfica de instituciones públicas que realizan distintas actividades. Este tipo de información se refiere básicamente a instituciones brasileñas. Los estudios se expresan en forma de páginas web que contienen mapas de Brasil en los que se recoge la localización de instituciones y actividades realizadas por las mismas en relación a materias concretas ${ }^{26}$.

La lista de materias en forma de temas generales en la actualidad es la siguiente: medio ambiente; cultura y educación pública; democracia, convergencia e inclusión digital; hacienda pública, poder judicial, ministerio público, modelos y proyectos de gobierno electrónico, salud pública y seguridad pública. En relación a cada tema se presentan varios mapas de ámbito federal o estatal, en los cuales, por ejemplo, se localiza la situación de instituciones, centros de estudio, actividades realizadas, distribución de recursos públicos con respecto a proyectos concretos, sitios web analizados siguiendo la metodología LEFIS.

Los mapas son resultados de investigaciones realizadas bajo la responsabilidad de investigadores concretos que elaboran trabajos o artículos sobre la respectiva materia. Pretenden crear discusión, a la vez que, fundamentalmente, ilustrar sobre las materias estudiadas a ciudadanos que no tengan formación especializada sino simplemente interés por conocer cuestiones concretas a efectos de crear opinión política. En este sentido son instrumentos tecnológicos que fomentan la democracia.

\section{Conclusiones}

Las expuestas en este trabajo son diferentes formas, concretas, de crear democracia "electrónica" mediante Internet y por ello son distintas posibilidades de incrementar la democracia. Las posibilidades muestran que aunque la democracia en Internet siempre estará limitada por el funcionamiento inevitablemente "interesado" de la gobernanza de Internet,

ew\&id=26\&Itemid=69>. Las páginas han sido accedidas el 15 de marzo de 2012.

26 Ver los mapas en: <http://egov.ufsc.br/portal/mapa\#mapabove>. La página ha sido accedida el 15 de marzo de 2012. 
hay margen para incrementarla, entendida ésta como participación ciudadana en el conocimiento de las actividades públicas propiciando al mismo tiempo la inclusión digital utilizando los recursos que contiene Internet.

\section{Referencias}

ALEXY, Robert. Begriff und geltung des rechts. Freiburg/München: Alber, 1992, p. 201-206.

ALVAREZ, R. Michael; HALL, Thad E. Electronic elections: the perils and promises of digital democracy. Princeton y Oxford, 2010.

CARBONELL, Miguel. Constitution's functionality and social rights: outline of some problems. Estudios Constitucionales, 6 (2), 43-71, 2008.

COUNCIL OF EUROPE. Ad hoc Committee on E-democracy, Council of Europe. Committee of Ministers. Electronic democracy ("e-democracy") $\operatorname{Recommendation} \mathrm{CM} / \operatorname{Rec}(2009) 1$ and explanatory memorandum. Strasbourg. 2009.

EHRLICH, Eugen. Freie Rechtsfindung und freie Rechtswissenschaft. Aalen : Scientia Verlag, 1987.

ENGISCH, Karl. La idea de concreción en el derecho y en la ciencia jurídica actuales. Pamplona: Navarra, 1968. 349-394.

ESSER, Josef. Principio y norma en la elaboración jurisprudencial del derecho privado. Barcelona: Bosch, 1961. p. 309-339.

GADAMER, Hans-Georg. Verdad y método: fundamentos de una hermeneútica filosófica. Salamanca: Sígueme,1977. 360 p.

GALINDO, Fernando. Democracia electrónica, Internet y gobernanza. Derecho y Tecnología, 109-125. 2011. In: GALINDO, F. Electronic democracy and governance. En Kleve, P. Van Noortwijk, (Eds.), Something bigger than yourself: Essays in honour of Richard de Mulder (41-56). Rotterdam: Erasmus University. 2011. 
. Justicia, gobernanza y legalidad. Seqüência - Estudos

Jurídicos e Políticos, Florianopólis, n. 55, p. 29-64, 2007.

HABERMAS, Jürgen. Facticidad y validez: sobre el derecho y el Estado democrático de derecho en términos de teoría del discurso. Madrid: Trotta, 1998. p. 208-237.

KATZ, Ellen D. Engineering the Endgame. Michigan Law Review, 109 (3), 349-386, 2010.

KELSEN, Hans. Teoría general del Estado. México: Nacional, 1979. p. 21-27.

MATURANA, Humberto. Self-consciousness: How? When? Where?

Constructivist Foundations, v. 1, n. 3, p. 91-102, 2006.

. EI árbol del conocimiento: las bases biológicas del

conocimiento humano. Madrid: Debate, 1990, p. 159-165.

MEZZAROBA, Orides; ROVER, Aires José. A urna eletrônica: sua contribuição para o aperfeiçoamento da democracia representativa partidária brasileira. In: GALINDO, Fernando; Rover, Aires José (Eds.). Derecho, gobernanza y tecnologías de la información en la sociedad del conocimiento, (63-73). Zaragoza: LEFIS Series 7, Prensas Universitarias. 2009.

PERELMAN, Chaim. La lógica jurídica y la nueva retórica. Madrid: Civitas, 1979. p. 176-233.

ROBLES, Gregorio. Teoría del derecho. 3. ed. Madrid: Civitas, 2010. VIEHWEG, Theodor. Topik und jurisprudenz. München: Beck, 1974. p. 111-119. 